\title{
REDUCING TWO PERSON, ZERO SUM GAMES WITH UNDERLYING SYMMETRY
}

\author{
K. R. PEARSON
}

(Received 13 February 1981)

Communicated by B. Mond

\begin{abstract}
We consider two person, zero sum games with several symmetries. Where such symmetries are present there is a group acting on the strategies of the game. We show how to use this action to produce a reduced game with a smaller matrix, but having the same value as the original game, and how to obtain optimal strategies for the original game from optimal strategies of the reduced game. An analysis of a simplified version of the popular game Mastermind is given to illustrate the theory developed.
\end{abstract}

1980 Mathematics subject classification (Amer. Math. Soc.): 90 D 05, 90 D 45.

The rules of many games give them a number of symmetries. When analysing such a game one keeps meeting cases which seem very much like ones already encountered and the natural approach is to try to link together somehow strategies that appear similar to each other.

In this paper we formalize these feelings for two person, zero sum games (with imperfect information) with several symmetries. Where such symmetries are evident, there is in fact a group acting on the strategies of the game. This action yields homogeneous optimal strategies (that is, optimal strategies in which linked strategies are used with equal probability) for each player, and makes it possible to guarantee the existence of a smaller matrix game with the same value as the original one. Further, all homogeneous optimal strategies in the original game can be obtained from a knowlege of all optimal strategies in the smaller or reduced game. The greater the number of symmetries, the smaller will this reduced game be, in general.

(c) Copyright Australian Mathematical Society 1982 
The reduction turns out to be the same as the one considered in [Gale/Kuhn/Tucker (1950), Application (e), pages 94-95], where they reduce games whose matrices can be partitioned into submatrices within each of which column totals are equal and row totals are equal. The virtue in considering the groups acting is that the recognition of such an action guarantees a priori that such a partitioning is possible (without first calculating the full matrix and having to play with rearrangements of its rows and columns) and indeed tells in advance exactly how to do the partitioning.

The reduction leads to the concept of a strategy being weakly dominated (see Section 2). Such a strategy may not be dominated in the usual sense but the results of Section 2 show that it can be ignored as its only contribution to the reduced matrix is dominated in the usual sense. In a simplified version of the popular game Mastermind (an analysis of which prompted the results of this paper) the original matrix is $9 \times 168$ and contains no row or column domination. However weak domination allows us to ignore 72 of player 2's strategies. Then reduction using the underlying symmetries, following the method developed here (the group involved is $S_{2} \times S_{3}$, where $S_{n}$ is the group of all permutations of $\{1,2, \ldots, n\})$, produces a $2 \times 3$ matrix which can of course be easily solved and used to find the value of the original game and to produce all homogeneous optimal strategies for it. More details are given in Section 3.

\section{Theory}

A group $G$ acts on a set $S$ when there is a function from $S \times G \rightarrow G$, mapping $(s, g) \rightarrow s g$ such that, for all $s \in S, g, h \in G$,

$$
s(g h)=(s g) h, \quad s 1=s
$$

where 1 is the identity of $G$. The action of a group $G$ on a set $S$ partitions $S$ into the orbits under $G$ : two elements $s_{1}, s_{2}$ of $S$ are in the same orbit if $s_{1} g=s_{2}$ for some $g \in G$ [see, for example, Mac Lane/Birkhoff (1979), pages 70-71].

We consider a finite, two person, zero sum game in normal form with pure strategies $\Gamma=\left\{\gamma_{1}, \ldots, \gamma_{m}\right\}$ for player 1 and $\Delta=\left\{\delta_{1}, \ldots, \delta_{n}\right\}$ for player 2 with payoff matrix $A=\left(a_{i j}\right)$ where $a_{i j}=\pi\left(\gamma_{i}, \delta_{j}\right)$ is the payoff to player 1 when the players use $\gamma_{i}$ and $\delta_{j}$ respectively. We suppose that there is a (finite) group $H$ (of symmetries) acting on both $\Gamma$ and $\Delta$ so that

$$
\pi(\gamma, \delta)=\pi(\gamma h, \delta h)
$$

for all $\gamma \in \Gamma, \delta \in \Delta, h \in H$; this is a formal way of stating that symmetrical positions have the same payoff. We denote by $X$ and $Y$ the sets of mixed strategies for players 1 and 2 respectively. 
First notice that the action of $H$ extends to all $m \times 1$ (or $n \times 1$ ) row vectors linearly; that is, if

$$
x=\left(x_{1}, \ldots, x_{m}\right)=x_{1} \gamma_{1}+\cdots+x_{m} \gamma_{m}
$$

and $h \in H$ then

$$
x h=x_{1}\left(\gamma_{1} h\right)+\cdots+x_{m}\left(\gamma_{m} h\right)
$$

For each $i, x h$ has its $i$ th component $(x h)_{i}$ equal to the $k$ th component $x_{k}$ of $x$ where $\gamma_{i} h^{-1}=\gamma_{k}$. If $x \in X$ and $h \in H$ then $x h \in X$ again. It follows readily from (1) that

$$
(x h) A=(x A) h \text { and } A(y h)^{T}=\left(A y^{T}\right) h
$$

for all $x \in X, y \in Y, h \in H$. (For example, if $\gamma_{i} h^{-1}=\gamma_{k(i)}$ for all $i$, the $j$ th component of $(x h) A$ equals

$$
\sum_{i}(x h)_{i} \pi\left(\gamma_{i}, \delta_{j}\right)=\sum_{i} x_{k(i)}\left(\gamma_{i} h^{-1}, \delta_{j} h^{-1}\right)=\sum_{i} x_{k(i)} \pi\left(\gamma_{k(i)}, \delta_{j} h^{-1}\right)
$$

which equals the $l$ th entry of $x A$ if $\delta_{j} h^{-1}=\delta_{l}$, and so equals the $j$ th entry of $(x A) h$.$) Since$

$$
(z h)(w h)^{T}=z w^{T}
$$

for all $h \in H$ and all $m \times 1$ (or $n \times 1$ ) vectors $z$ and $w$, we conclude that, for all $x \in X, y \in Y, h \in H$,

$$
(x h) A(y h)^{T}=x A y^{T} \text {. }
$$

Let $h \in H$. Since $x \mapsto x h(y \mapsto y h)$ is a bijection from $X(Y)$ to itself, the following is an easy consequence of (2).

LEMMA 1. Let $x \in X, y \in Y, h \in H$. Then $(x, y)$ is an equilibrium pair if and only if $(x h, y h)$ is. Thus $x$ is optimal for player 1 if and only if $x h$ is.

Let $H=\left\{h_{1}, \ldots, h_{r}\right\}$. For each $x \in X$ we define $\bar{x} \in X$ by

$$
\bar{x}=\left(\sum_{h \in H} x h\right) / r \text {. }
$$

Since $\bar{x}$ is a convex linear combination of $x h_{1}, \ldots, x h_{r}$ it follows that $\bar{x}$ is optimal for player if $x$ is. Notice also that $\bar{x}$ is homogeneous in the sense that if $\gamma_{i}$ and $\gamma_{k}$ are in the same orbit of $\Gamma$ under $H$ (that is $\gamma_{k}=\gamma_{i} h$ for some $h \in H$ ) then the $i$ th and $k$ th components of $\bar{x}$ are equal; this is an easy exercise. We denote by $\bar{X}$ the set of all homogeneous elements of $X$; clearly the map $x \mapsto \bar{x}$ maps $X$ onto $\bar{X}$.

LEMMA 2. If $x \in X$ is optimal for player 1 then so is $\bar{x}$, and $\bar{x} \in \bar{X}$. 
Let $\Gamma_{1}, \ldots, \Gamma_{s}$ be the orbits of $\Gamma$ under $H$, suppose that these have sizes $m_{1}, \ldots, m_{s}$ respectively, and suppose that the $\gamma$ 's are renamed so that $\gamma_{1} \in$ $\Gamma_{1}, \ldots, \gamma_{s} \in \Gamma_{s}$. For each $x \in \bar{X}$ we define an $s \times 1$ vector

$$
d(x)=\left(m_{1} x_{1}, \ldots, m_{s} x_{s}\right) .
$$

Since $x$ has $m_{1}$ entries equal to $x_{1}$ etc., $d(x)$ is a probability vector. If $x \in \bar{X}$, we see that, for all $j$, the $j$ th entry of $x A$ equals

$$
x_{1} \sum_{\gamma_{k} \in \Gamma_{1}} a_{k j}+\cdots+x_{s} \sum_{\gamma_{k} \in \Gamma_{s}} a_{k j}
$$

which equals the $j$ th entry of $d(x) B$ where $B=\left(b_{i j}\right)$ is the $s \times n$ matrix with

$$
b_{i j}=\frac{1}{m_{i}} \sum_{\gamma_{k} \in \Gamma_{i}} a_{k j}
$$

for all $i, j$. Thus if $x \in \bar{X}$,

$$
x A=d(x) B .
$$

Let $X_{1}$ denote the set of $s \times 1$ probability vectors. Then $d: \bar{X} \rightarrow X_{1}$ is a bijection with inverse $e: X_{1} \rightarrow \bar{X}$ where $e\left(x_{1}, \ldots, x_{s}\right)=\left(z_{1}, \ldots, z_{m}\right)$ with $z_{i}=$ $x_{k} / m_{k}$ when $\gamma_{i} \in \Gamma_{k}$. It follows that, for all $x \in X_{1}$,

$$
x B=e(x) A .
$$

The following is a simple consequence of (3) and (4).

THEOREM 3 (Reduction with respect to player 1). Let $B$ be obtained from $A$ and $H$ as above. Then

(i) $x \in \bar{X}$ is optimal in $A$ if and only if $d(x)$ is optimal in $B$,

(ii) if $y \in Y$ is optimal in $A$ then it is optimal in $B$, and

(iii) the values of $A$ and $B$ are equal.

Thus if we find an optimal $x$ for player 1 in $B$, we obtain from it $e(x)$ which is optimal for player 1 in $A$, and all homogeneous optimal strategies for player 1 in $A$ arise in this way. (However player 1 may also have nonhomogeneous optimal strategies in $A$.)

A very simple example of this reduction is Colonel Blottowith 6 companies for 2 locations against the enemy's 5 companies where each side must station at least one company in each location (see [Williams (1966) pages 159-163]). Here equal forces in any location is a draw and a superior force overwhelms the opposition 
and the payoff equals the size of the force overwhelmed. The payoff matrix $A$ is

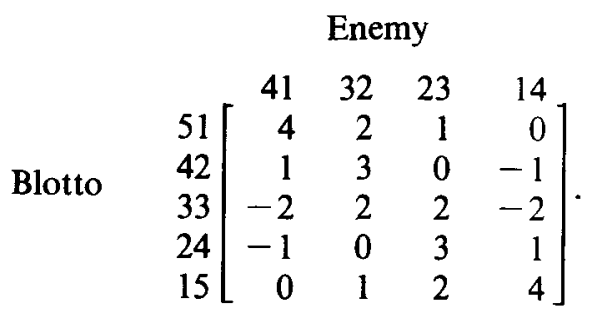

Here 42 means 4 companies in location 1 and 2 in location 2 . The symmetry here is between the two locations, which are essentially the same so that the group $H$ is the group $S_{2}$ of permutations of the two locations. Clearly condition (1) holds. The orbits of $\Gamma$ are $\{51,15\},\{42,24\},\{33\}$ and the reduced matrix $B$ is

$$
\begin{gathered}
\{51,15\} \\
\{32,24\}
\end{gathered}\left[\begin{array}{rrrr}
41 & 32 & 23 & 14 \\
2 & \frac{3}{2} & \frac{3}{2} & 2 \\
0 & \frac{3}{2} & \frac{3}{2} & 0 \\
-2 & 2 & 2 & -2
\end{array}\right] .
$$

Of course $H$ also acts on $\Delta$ and the orbits are $\{41,14\},\{32,23\}$. Notice that any two strategies in the same orbit of $\Delta$ have equal columns in $B$. This always happens.

Lemma 4. Suppose $\delta_{1}, \delta_{2} \in \Delta$ are in the same orbit under $H$. Then in the matrix $B$ they have equal columns.

Proof. Let $h \in H$ be such that $\delta_{2}=\delta_{1} h$. The $i$ th entry in the $\delta_{2}$-column of $B$ is

$$
\frac{1}{m_{i}} \sum_{\gamma \in \Gamma_{i}} \pi\left(\gamma, \delta_{1} h\right)=\frac{1}{m_{i}} \sum_{\gamma \in \Gamma_{i}} \pi\left(\gamma h^{-1}, \delta_{1}\right)
$$

by (1). But $\left\{\gamma h^{-1} \mid \gamma \in \Gamma_{i}\right\}=\Gamma_{i}$ and so this equals the $i$ th entry in the $\delta_{1}$-column of $B$.

Thus while $B$ is an $s \times n$ matrix it only has at most $t$ distinct columns, where $\Delta_{1}, \ldots, \Delta_{t}$ are the orbits of $\Delta$ under $H$. By using column dominance (or applying the analogous reduction with respect to player 2 to $B$ ) we obtain an $s \times t$ matrix $D$ with the same value as $A$ and with a one-to-one correspondence (via the functions $d$ and $e$ ) between the homogeneous optimal strategies for player 1 in $A$ and all optimal strategies for player 1 in $D$. The entry $d_{i j}$ in row $\Gamma_{i}$ and column $\Delta_{j}$ 
of $D$ is given by

$$
d_{i j}=\frac{1}{m_{i}} \sum_{\gamma \in \Gamma_{i}} \pi(\gamma, \delta)
$$

for any $\delta \in \Delta_{j}$.

Of course we could first reduce via the second player to an $m \times t$ matrix $C$. Then two rows in the same orbit of $\Gamma$ are equal and so we could obtain from $C$ an $s \times t$ matrix $D^{\prime}$, which we could use to find all homogeneous optimal strategies for player 2 in $A$. The entry $d_{i j}^{\prime}$ in row $\Gamma_{i}$ and column $\Delta_{j}$ is given by

$$
d_{i j}^{\prime}=\frac{1}{n_{j}} \sum_{\delta \in \Delta_{j}} \pi(\gamma, \delta)
$$

for any $\gamma$ in $\Gamma_{i}$ (where $\left|\Delta_{j}\right|=n_{j}$ ). But since (6) is independent of $\delta \in \Delta_{j}$ and (7) is independent of $\gamma \in \Gamma_{i}$ we have

$$
d_{i j}=\frac{1}{m_{i} n_{j}} \sum_{\delta \in \Delta_{j}} \sum_{\gamma \in \Gamma_{i}} \pi(\gamma, \delta)=d_{i j}^{\prime}
$$

Thus $D$ and $D^{\prime}$ are equal, and so we can use $D$ to find all homogeneous optimal strategies for either player.

TheOREM 5. Let $D$ be the $s \times t$ matrix obtained as above. Then $D$ has the same value as $A$ and the sets of homogeneous optimal strategies for the players in $A$ can be obtained by applying the appropriate function e to the sets of all optimal strategies in D.

In the Blotto example above, $D$ is

$$
\begin{gathered}
\{51,15\} \\
\{42,24\} \\
\{33\}
\end{gathered}\left[\begin{array}{cc}
\{41,14\} & \{32,23\} \\
2 & \frac{3}{2} \\
0 & \frac{3}{2} \\
-2 & 2
\end{array}\right]
$$

This has value $14 / 9$ with unique optimal strategies $(8 / 9,0,1 / 9)$ and $(1 / 9,8 / 9)$. Thus the original game $A$ has value $14 / 9$ and the unique homogeneous optimal strategies in $A$ are

$$
\begin{aligned}
& x=(4 / 9,0,1 / 9,0,4 / 9), \\
& y=(1 / 18,4 / 9,4 / 9,1 / 18) .
\end{aligned}
$$

(The nonhomogeneous $(3 / 90,48 / 90,32 / 90,7 / 90)$ is also optimal for player 2 [Williams (1966) page 162].) 
The matrix $A$ reflects the symmetries better if it is rewritten with strategies in the same orbit adjacent.

$$
\begin{array}{r}
51 \\
15 \\
42 \\
24 \\
33
\end{array}\left[\begin{array}{rr:rr}
41 & 14 & 32 & 23 \\
4 & 0 & 2 & 1 \\
0 & 4 & 1 & 2 \\
\hdashline 1 & -1 & 3 & 0 \\
\hdashline-1 & 1 & 0 & 3 \\
\hdashline-2 & -2 & 2 & \frac{2}{2}
\end{array}\right]
$$

In each of the 6 submatrices column sums are equal, as are row sums (but the common column sum is not necessarily the same as the common row sum as the $2 \times 1$ matrices show). Again it follows easily from (1) that this always happens. (The proof is essentially given in Lemma 4.)

LEMMA 6. If $\Gamma_{i}$ and $\Delta_{j}$ are orbits in $\Gamma$ and $\Delta$ respectively then the submatrix of $A$ relevant to $\Gamma_{i}$ and $\Delta_{j}$ has all row sums equal and all column sums equal.

Thus if we rearrange the rows and columns into their orbits, the resulting partition is as in Application (e), pages 94-95 of [Gale/Kuhn/Tucker (1950)]. Knowing that a group of symmetries is acting on a game makes it possible to tell what this partitioning is without writing down the matrix in full, and usually makes it unnecessary to write down more than a few of the columns (or rows) in order to calculate its value and find optimal strategies. We illustrate this with the example in Section 3.

\section{Weak domination}

Consider two pure strategies $\gamma_{1}, \gamma_{2}$ for player 1. We say that $\gamma_{1}$ is weakly dominated by $\gamma_{2}$ if

(i) $\gamma_{1}$ and $\gamma_{2}$ are not in the same orbit of $\Gamma$ under $H$, and

(ii) in the matrix $C$ obtained via the reduction with respect to player 2 , the $\gamma_{1}$-row is dominated in the usual sense by the $\gamma_{2}$-row.

Condition (ii) is the same as requiring that, for all orbits $\Delta_{i}$ of $\Delta$ under $H$,

$$
\sum_{\delta \in \Delta_{i}} \pi\left(\gamma_{1}, \delta\right) \leqslant \sum_{\delta \in \Delta_{i}} \pi\left(\gamma_{2}, \delta\right) .
$$

(Notice also that if the domination in (ii) is strict, that is the two rows are not equal, condition (i) automatically holds as strategies in the same orbit have equal rows in $C$, by Lemma 4.) 
If $\gamma_{1}$ in orbit $\Gamma_{1}$ is weakly dcminated by $\gamma_{2}$ in orbit $\Gamma_{2}$, the $\Gamma_{1}$-row of $D$ is dominated by the $\Gamma_{2}$-row of $D$ and so there is an optimal strategy for player 1 in $D$ in which $\Gamma_{1}$ is not used. Thus, by Theorem 5 , there is a homogeneous optimal strategy for player 1 in $A$ in which $\gamma_{1}$ (and all strategies in $\Gamma_{1}$ ) are not used. Thus we can ignore strategies which are weakly dominated.

For example, in the Blotto example above, the 51 and 42 rows of $C$ are $(2,3 / 2)$ and $(0,3 / 2)$ respectively. Thus 42 is weakly dominated by 51 and so we can ignore 42 (and therefore 24). Notice however that 42 is not dominated in the usual sense by 51 in $A$.

\section{An example}

In this section we examine a simplified version of the popular game Mastermind and illustrate how the reduction techniques in $\$ 1$ and the notion of weak dominance enable us to transform this to a manageable problem.

Player 1, the "coder", selects a "code" which is an ordered pair of pegs each of which is one of the 3 colours red $(R)$, blue $(B)$ or green $(G)$. Thus the coder has $3^{2}=9$ pure strategies, namely $R R, R B, R G, B R, B B, B G, G R, G B, G G$. Player 2, the "decoder", makes successive guesses, trying to identify the code selected. After each guess he is told how many pegs $s_{1}$ in his guess are exactly right (correct colour in correct place) and how many pegs $s_{2}$ are partly right (correct colour but in the wrong place); we call this information the "signal" $\left(s_{1}, s_{2}\right)$. The payoff to the coder is the number of guesses it takes the decoder to identify the code. (In the two commerically available versions a code consists of four or five pegs each of which is one of six or eight colours.)

There are two kinds of symmetry here, one obtained by permuting the 3 colours (this is the group $S_{3}$ ) and the other (the group $S_{2}$ ) by permuting the order of the pegs in a code (for example mapping $B G$ to $G B$ ). It is easy to see that the action of these two groups commute with each other (that is $\gamma g h=\gamma h g$ for a strategy $\gamma$ and for $\left.g \in S_{3}, h \in S_{2}\right)$; thus indeed their direct product $H=S_{3} \times S_{2}$ acts on this game. There are two orbits for the coder, namely $\Gamma_{1}=\{R R, B B, G G\}$ and $\Gamma_{2}=\{R B, R G, B R, B G, G R, G B\}$.

The decoder has a large number of different pure strategies. A strategy for him consists of an initial guess then, for each possible signal, a second guess etc. For example, if he picks $R B$ as his first guess there are only two signals $(0,1)$ and $(1,0)$ which leave him in any doubt as to the code. Let $\delta_{1}$ denote the strategy where he picks $B G$ if the signal is $(0,1)$ and $R G$ if it is $(1,0)$ (after which he knows the code 
and proceeds accordingly); we picture this as

$$
\begin{aligned}
& \delta_{1}=R B \frac{(0,1)}{(1,0)}_{R G} B G \\
& \text { guess } 1 \text { guess } 2
\end{aligned}
$$

A detailed analysis of the decoder's pure strategies shows that there are 168 undominated ones. Thus the matrix $A$ is $9 \times 168$ and it would be a long and tedious task to write out $A$ in full, let alone use it to find the value of the game. The decoder's 168 undominated strategies are of one of five types:

(i) several yielding the same reduced column as $\delta_{1}$ (indeed there are 24 in two orbits, but it is not necessary to know this in order to solve the game),

(ii) several (indeed 24 in two orbits) yielding the same reduced column as

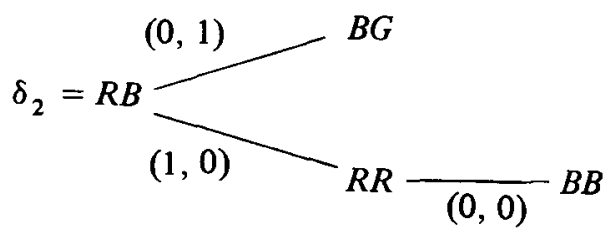

guess 1 guess 2 guess 3

(iii) several (24 in two orbits) yielding the same reduced column as

$$
\delta_{3}=R B \frac{(0,1)}{(1,0)} B G
$$

(iv) 48 in four orbits yielding the same reduced column as

$$
\delta_{4}=R R \frac{(1,0)}{(0,0)}^{\frac{(1,0)}{(1,0)}} B G
$$

(v) 48 in four orbits yielding the same reduced column as

$$
\delta_{5}=R R \underbrace{(1,0)}_{(0,0)} B B
$$


The corresponding columns in the matrix $B$ obtained by a first player reduction are

$$
\begin{aligned}
& \begin{array}{lllll}
\delta_{1} & \delta_{2} & \delta_{3} & \delta_{4} & \delta_{5}
\end{array}
\end{aligned}
$$

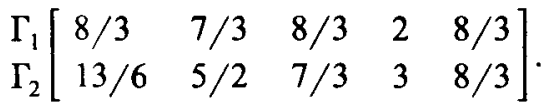

Thus $\delta_{5}$ and $\delta_{3}$ are weakly dominated by $\delta_{1}$ and we can ignore these columns. Thus, using Theorem 5 , we are led to consider the matrix

$$
\left[\begin{array}{ccc}
8 / 3 & 7 / 3 & 2 \\
13 / 6 & 5 / 2 & 3
\end{array}\right]
$$

(Actually $D$ is obtained from this matrix by repeating the first and second columns twice for the two orbits in each of (i) and (ii) above and by repeating the third column four times.) This $2 \times 3$ game is easy to solve and we find it has value $29 / 12$ and (unique) optimal strategies $(1 / 2,1 / 2)$ and $(1 / 4,3 / 4,0)$. Thus the value of this form of Mastermind is $29 / 12$ and we can read off homogeneous optimal strategies. The coder uses each of his strategies in $\Gamma_{1}$ with probability $1 / 6$ and each strategy in $\Gamma_{2}$ with probability $1 / 12$. (Indeed it is not difficult to see by a separate calculation that this is the only optimal strategy for the coder.) The decoder has infinitely many homogeneous optimal strategies because of the different orbits giving rise to equal columns. For example, one is where he uses each of the twelve strategies in the orbit of $\delta_{1}$ with probability $1 / 48$ and each of the twelve in the orbit of $\delta_{2}$ with probability $1 / 16$.

\section{References}

D. Gale, H. W. Kuhn and A. W. Tucker (1950), 'Reduction of game matrices', Contributions to the theory of games, vol. 1, pp. 89-96 (Ann. of Math. Studies, no. 24, Princeton Univ. Press, Princeton, N.J.).

S. Mac Lane and G. Birkhoff (1979), Algebra (2nd edition, Macmillan, New York).

J. D. Williams (1966), The compleat strategyst (McGraw-Hill, New York).

Department of Mathematics

La Trobe University

Bundoora, Victoria 3083

Australia 